\title{
The effect of area, separation, and dichoptic presentation on the perception of achromatic color
}

\author{
WILLIAM R. WHIPPLE \\ University of Arkansas at Little Rock, Little Rock, Arkansas \\ HANS WALLACH \\ Swarthmore College, Swarthmore, Pennsylvania \\ and \\ FREDERICK J. MARSHALL \\ Harvard University School of Medicine, Boston, Massachusetts
}

\begin{abstract}
In a center-surround arrangement of two luminances, luminance ratio, area of the surrounding higher luminances, and separation of the two luminances were varied. Decreasing the area of the surrounding luminance caused the achromatic color in the center to be lighter, and a similar but smaller effect was caused by increasing separation up to approximately $30^{\prime}$ of visual an. gle; increasing separation beyond $30^{\prime}$ did not lighten the color further. At higher luminance ratios, which resulted in darker colors, the effect of decreasing the area of the surround became more pronounced. Dichoptic presentation of the surround and center luminances resulted in perception of achromatic colors in the center that depended on luminance ratios, in a manner similar to perception of binocularly presented luminances. Dichoptically produced colors were somewhat lighter than those that resulted when center and surround were given to each eye.
\end{abstract}

When a light-emitting area is viewed against a dark background, it appears luminous. If, however, two areas with different luminances are juxtaposed, neither area appears luminous; rather, both are perceived as more or less opaque achromatic surface colors, the brighter area appearing white and the area of lower luminance as a shade of gray. The colors thus produced are known as induced colors, since the color of each area is induced by the presence of the other luminance. Although the phenomenon may be observed with a number of different displays, the most common is a center-surround arrangement, in which a central area of one luminance is surrounded by an area of a different (usually higher) luminance. Using this arrangement, Wallach (1948) obtained a variety of shades of gray in the central area and showed that the particular shade perceived depended not on the luminance of the central area itself, but on the ratio between the luminances of the center and surround. This accounts for the phenomenon of color constancy, which results in the correspondence between perceived colors and reflectances even when illumination changes. The luminances that reach the eyes vary not only with reflectance but also with illumination. However, when illumination is uniform, the

This work was supported by Grant BSN-8318772 from the National Science Foundation to Swarthmore College, Hans Wallach, principal investigator. All correspondence should be sent to Hans Wallach, Department of Psychology, Swarthmore College, Swarthmore, PA 19081. luminances stand in fixed ratios to each other, and these ratios depend on the ratios of the reflectances. If the perceived colors depend on the ratios between luminances, correspondences between perceived colors and reflectances result, and perceived achromatic colors are veridical.

Where in the visual processing system color induction occurs is still uncertain. Cornsweet $(1970$, p. 371$)$ proposed a mechanism for brightness constancy that takes place very early, probably at the retinal level. It is possible that color induction relies in part on retinal mechanisms such as this. Land and McCann (1971) discussed achromatic color in terms of rank orderings of all brightnesses that exist in conceptual fields called retinexes. These retinexes are not identified with any particular physiological mechanism; rather, they are hypothetical constructs that explain the phenomena of both achromatic and chromatic color perception.

To learn more about the nature of color induction, one can examine what variations in stimulus configuration affect the colors perceived. A systematic study of several variables was performed by Newson (1958). Experiments resembling those of Gelb (1932) and Wallach (1948) showed that an area of low luminance can be seen as light gray or white when it is seen in a much darker surround, but would become a dark gray when a much higher luminance is introduced. Newson also confirmed Wallach's 
finding that the shade of gray perceived could be altered by changing the luminance of the inducing field, and showed that the highest luminance in view was of primary importance in determining the target color. In addition, he varied the separation between target and inducing surfaces, showing that induction falls off rapidly as this separation increases up to a few minutes of visual angle, but much more slowly with a greater increase in separation. Newson also varied the area of an inducing field that surrounded the target, and found that when the area of the surround was less than approximately $20 \%$ of the area of the target, the induction effect diminished sharply.

Newson's (1958) experiments are valuable because the dependent variable in each case was the subject's selection from a Munsell chart of the shade of gray that matched the target's apparent color. Other studies (e.g., Leibowitz, Mote, \& Thurlow, 1953, for separation; Cole \& Diamond, 1971, for separation and for area of surround) have used brightness-matching techniques, which do not isolate the subject's perception of color. However, Newson's experiments have several limitations: (1) The stimuli consisted of gray papers of various reflectances illuminated by a $500-\mathrm{W}$ lamp; this limited the obtainable luminance ratios between inducing and test areas to a maximum of about 5:1. (2) Owing to the nature of his experimental setup, Newson did not attempt to specify all of the "photometric brightness relationships" existing in the visual field. For example, the stimuli were observed in a large room with black-painted walls and black cloth hangings serving as a backdrop for the targets; this means that the targets were surrounded by an area of low but visible luminance that could interact with the target and inducing surfaces. That such interactions did indeed take place can be seen from the fact that grays of various values did not look equally white when viewed without an inducing luminance present. (Indeed, in the total absence of background luminance these targets would not have looked light gray or white, but would have been luminous - that is, they would have appeared to emit light.) (3) Except in the experiment in which area of surround was varied, Newson's inducing fields were squares located alongside the square target luminances. Although induction can be obtained with this configuration, it is weaker than in a center-surround display. (4) Interpretation of Newson's results on separation of inducing and test field is complicated by the fact that his apparatus did not illuminate the inducing field equally (from the subject's vantage point) at all separations used.

In the experiments reported here, we attempted to replicate and extend Newson's findings, as well as the earlier data of Wallach (1948), in a situation that allowed precise control of the luminance relationships present in the stimulus array, and that permitted the use of luminance ratios greater than those available to Newson. Using a center-surround display, we examined the effects on subjects' choices of matching Munsell grays of variations in luminance ratio, in area of surround, and in separation between center and surround. We also introduced another variable, which had not previously been studied: the effect of presenting the two interacting areas of luminance dichoptically, that is, of presenting the central disk-shaped target to one eye and the ring-shaped surround to the other eye. This arrangement is particularly valuable for determining whether the interaction that yields induced color takes place in the retina or at a more advanced level of visual processing.

In planning the dichoptical arrangement, we had to consider ways in which to avoid binocular rivalry, which is very strong when the inner edge of the ring-shaped surround and the edge of the disk-shaped target fall on circles of corresponding points on the two retinas. In one eye the luminance in the disk borders on the dark surround, and in the other eye the luminance in the ring forms the surround of a dark region inside the ring. In such a case, contours with reversed luminance differences would have to be binocularly fused-but contours with such reversed luminance differences do not fuse. To avoid the resulting rivalry, the inner edge of the ring and the outer edge of the disk would have to be separated by a narrow dark region. To achieve this, we constructed our dichoptical arrangement so that the diameter of the inner edge of the ring was larger than the diameter of the outer edge of the disk. When the monocular view of the disk and the monocular view of the ring were properly combined, the dark region formed a narrow ring that separated the two dichoptically incompatible contours. ${ }^{1}$ We called this narrow dark ring the annulus. To find out how it affected induction, we varied its width, and we also investigated the effect it had in binocular presentation.

\section{EXPERIMENT 1}

In Experiment 1 we measured the values of the grays induced in a central disk surrounded by rings of various widths. Subjects selected from a Munsell chart sample chips whose color appeared to match that of the central disk; this is the technique that Newson (1958) employed. The ring always had a higher luminance than the disk, and its width was varied in three steps. Each of these three spatial conditions was presented with three luminance ratios between ring and disk, for a total of nine conditions.

\section{Method}

Subjects. Eight undergraduate students, who were paid for their services, participated.

Equipment. The ring-and-disk display was obtained by combining ring-shaped and disk-shaped images by means of a half-silvered mirror. The subject looked through two eyeholes into a large wooden box fitted with a headrest. The box had two square openings, covered by translucent screens that could be illuminated from outside the box. One opening was on the face of the box opposite the subject's station; the other was on the subject's left. A black cardboard mask with a circular aperture was placed just in front of the screen on the left; masks with ring-shaped apertures could be inserted in front of the other screen. A half-silvered mirror was mounted at an angle in the subject's line of sight so that the image from the circular aperture could be centered in the ring-shaped one; the subject could adjust the angle of the mirror slightly to obtain perfect align- 
ment of the two images. The optical distance from the eyeholes to either aperture was $80 \mathrm{~cm}$. The screens were illuminated by Kodak Carousel projectors located outside the box. The light from the projectors was passed through pale blue filters that neutralized the slight yellowish tint of the incandescent projector light. A series of neutral density filters, mounted on slides, could be inserted in the projectors to vary the intensities of their beams. The interior of the box was lined with black velvet.

A second, smaller box was placed to the left of the subject. Opposite the eyeholes in this box, at a distance of $76 \mathrm{~cm}$, was a 32 step Munsell achromatic color chart, consisting of chips that reflected from $90 \%$ to $2.5 \%$ of incident light in steps of 0.25 Munsell value. The chips were visible through cutouts in the white paper covering the chart, and were identified by numbers printed on that paper. The chart was illuminated by both fluorescent and incandescent lamps inside the box; the relative intensities of these sources were adjusted so that the color temperature of the light inside the Munsell box matched that of the larger box. When selecting color matches the subject would look alternately into the stimulus box and into the Munsell box.

To prevent the subject from dark-adapting between trials, the experimental room had a white ceiling and white walls, illuminated by a lamp that directed the light of a $100-\mathrm{W}$ bulb toward the ceiling. The outer walls of the two light boxes, which the subject faced, were painted white. Luminance of the walls in the experimental room was measured at $0.43 \mathrm{~cd} / \mathrm{m}^{2}$. All luminance measurements were made with a Macbeth illuminometer positioned at the subject's station.

Stimuli. The diameter of the disk was always $6.35 \mathrm{~cm}$, subtending $4^{\circ} 33^{\prime}$ of visual angle. The inner diameter of each of the rings was $6.5 \mathrm{~cm}$; thus the annulus between ring and disk was $0.08 \mathrm{~cm}$ wide and subtended $3^{\prime}$ of visual angle. The three rings were 6.9 , 8.2 , and $10.1 \mathrm{~cm}$ in diameter; thus, the widths of the rings were $0.20,0.85$, and $1.8 \mathrm{~cm}$, subtending $9^{\prime}, 36^{\prime}$, and $1^{\circ} 17^{\prime}$ of visual angle, respectively.

The luminance of the disk was always $36.8 \mathrm{~cd} / \mathrm{m}^{2}$. The luminance of the ring varied, being $471.1,296.3$, or $135.3 \mathrm{~cd} / \mathrm{m}^{2}$. This made the ring:disk luminance ratios close to $13: 1,9: 1$, and $3.75: 1$.

Procedure. The subject began by adjusting the mirror so that a sample ring-and-disk display appeared properly aligned, that is, so that the ring and disk were concentric, with the annulus uniform in width. The experimenter then explained that the subject was to look back and forth between the stimulus display and the Munsell chart until he/she could select a color from the chart that matched the color of the disk

Measurements for Experiments 1 and 2 (discussed below) were taken together. There were three conditions in which the width of the ring was varied (Experiment 1) and three in which annuli of varying widths separated the disk from a ring of constant outer diameter (Experiment 2). These six conditions were presented first with one luminance ratio and subsequently with the second and third. The order of the six conditions in connection with each luminance ratio was random and varied across ratios, whereas the order of the ratios changed from subject to subject. Each of these 18 conditions was presented three times, with the order of arrangements randomized within subjects and the order of ratios randomized across subjects. The average of the reflectances of the color chips chosen by a subject on the three trials within any condition became that subject's score for that condition.

\section{Results and Discussion}

The mean reflectances of the matching color chips chosen by the subjects are listed in Table 1 . The averages shown at the foot of each column represent the average color matches for each luminance ratio. Averages for each of the three ring widths are shown in the right-hand column.
Table 1

Mean Reflectances of Matching Colors for Three Ring Widths and Three Luminance Ratios

\begin{tabular}{ccccc}
\hline & \multicolumn{3}{c}{ Luminance Ratio } & \\
\cline { 2 - 4 } Ring Width & $13: 1$ & $8: 1$ & $3.75: 1$ & Average \\
\hline $0.20 \mathrm{~cm}$ & 37.12 & 42.47 & 48.18 & 42.59 \\
$0.85 \mathrm{~cm}$ & 30.56 & 35.10 & 46.27 & 37.31 \\
$1.80 \mathrm{~cm}$ & 27.77 & 33.05 & 42.21 & 34.35 \\
Average & 31.82 & 36.88 & 45.56 & \\
\hline
\end{tabular}

As was expected, the matching colors became lighter as the luminance ratio decreased. This effect was highly significant $[F(2,14)=44.39, p<.001]$. The numbers in the right-hand column also show that the colors became darker as the ring became wider; this effect was also significant $[F(2,14)=33.76, p<.001]$. There was a significant statistical interaction between the two independent variables $[F(4,28)=3.98, p<.05]$, which reflected the fact that as the luminance ratio became smaller, the effect of ring width was diminished.

Our finding that increasing the area of an inducing surround causes the color of a central field to appear darker is broadly consistent with Newson's (1958) report for similar arrangements. Newson used only one luminance ratio (approximately 5:1), and surrounds whose areas ranged from $2 \%$ to $60 \%$ of the area of the central field. However, in Newson's experiment the induction effect reached an asymptotic value when the surround was approximately $28 \%$ of the area of the target; increasing the surround to $60 \%$ of the target's area had little additional effect. This result led Newson to conclude that the area immediately adjacent to the target is most effective in color induction, and that areas more widely separated from the target have little influence on the color of the target.

Our findings allow a different conclusion. The luminance ratio in our experiment that was closest to that used by Newson was 3.75:1 and for that ratio, variations in the width of the surround over a range from $13 \%$ to $148 \%$ of the target size had only a small effect on the appearance of the target field. However, at greater luminance ratios, increases in the area of the surround had progressively greater influence on the appearance of the target. As reported above, this interaction was statistically significant.

It should also be noted that our results were obtained with a dark annulus separating the target from the surround. The width of this annulus subtended $3^{\prime}$ of visual angle; thus the critical area immediately adjacent to the target was entirely dark. Despite this, robust color induction was obtained. In Experiment 2 we systematically examined the effects of separation between target and surround.

\section{EXPERIMENT 2}

Annuli of varying widths were introduced between the central target and the surrounding ring. In all cases the ring was $1.8 \mathrm{~cm}$ wide (the widest ring used in Experiment 1); the annulus width was varied by making the central disk progressively smaller. 


\section{Method}

Three disks, with diameters of $6.00,5.44$, and $4.40 \mathrm{~cm}$, were presented in conjunction with a wide surrounding ring (outer diameter $10.1 \mathrm{~cm}$, inner diameter $6.5 \mathrm{~cm}$, width of ring $1.8 \mathrm{~cm}$ ). This resulted in dark annuli of varying widths: $0.25,0.53$, and $1.05 \mathrm{~cm}$, subtending $11^{\prime}, 23^{\prime}$, and $52^{\prime}$ of visual angle, respectively. Luminance ratios were the same as for Experiment 1. As explained above, measurements for the two experiments were taken at the same time. Therefore, data from the conditions in Experiment 1 that used an annulus of $0.08 \mathrm{~cm}$ ( $3^{\prime}$ of visual angle) may be combined with data from Experiment 2 , so that the design is effectively $3 \times 4-$ three luminance ratios and four separations. As before, the dependent variable is the average of the percent reflectance of the color chips selected by each subject on three trials.

The subjects were the same as those used in Experiment 1.

\section{Results and Discussion}

Table 2 gives the results in the same format as Table 1, except that in this case the different rows refer to the four annulus widths. Thus, the differences between rows indicate the effect of increasing separation between the disk and the surrounding ring.

The averages of the mean color matches for the three luminance ratios closely resemble the corresponding data from Experiment 1: smaller luminance ratios led to lighter perceived colors. This effect is highly significant $[F(2,14)$ $=63.77, p<.001]$. Annulus width also had a significant effect on color judgments $[F(3,21)=3.61$, $p<.05]$. This separation effect is presented graphically in Figure 1, which shows that the effect of separation on color matches was greatest at small separations; beyond approximately $30^{\prime}$ of visual angle, the effect diminished greatly. The interaction between separation and luminance ratio was not significant $[F(6,42)=0.58, p>.05]$.

Our results show that induced achromatic colors become lighter as the target and the surround are separated, but that this effect decreases as the separation becomes larger; we found little difference in the apparent color of the target at separations of $23^{\prime}$ and $52^{\prime}$ of visual angle. Similar results have been reported before. Newson (1958) varied the separation of two square targets and found that introduction of a $6^{\prime}$ gap betwen them made the apparent color of the target with the lower luminance much lighter than when the two targets were adjacent. Increasing the separation further, up to $65^{\circ}$, had an effect that was much less pronounced, and Newson noted that the slight changes that were observed may have been artifacts of the ex-

Table 2

Mean Reflectances of Matching Colors for Four Annulus Widths and Three Luminance Ratios

\begin{tabular}{lcccc}
\multicolumn{3}{c}{ Annulus Widths and Three Luminance Ratios } \\
\cline { 2 - 4 } & \multicolumn{3}{c}{ Luminance Ratio } & \\
Annulus Width & $13: 1$ & $8: 1$ & $3.75: 1$ & Average \\
\hline $0.08 \mathrm{~cm} \mathrm{(3')}$ & 27.77 & 33.05 & 42.21 & 34.35 \\
$0.25 \mathrm{~cm}\left(11^{\prime}\right)$ & 28.33 & 34.57 & 43.98 & 35.63 \\
$0.53 \mathrm{~cm} \mathrm{(23})$ & 29.49 & 35.73 & 46.22 & 37.15 \\
$1.05 \mathrm{~cm}\left(52^{\prime}\right)$ & 31.19 & 36.65 & 45.30 & 37.71 \\
Average & 29.19 & 35.00 & 44.43 & \\
\hline
\end{tabular}

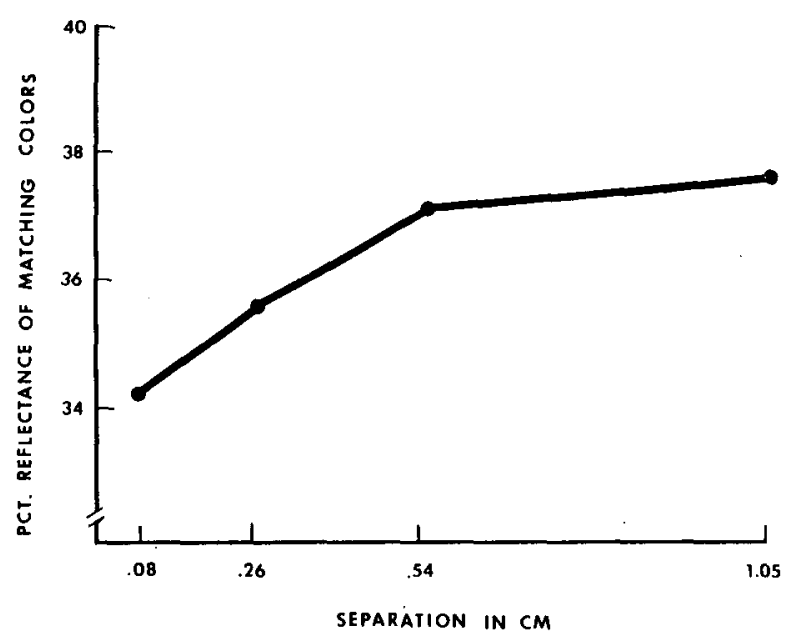

Figure 1. Mean color matches as a function of separation in centimeters.

perimental setup. Leibowitz et al. (1953) also varied separation between an inducing and a test field; their measure was the subject's adjustment of a reference field to provide a match with the test field. Although their procedure differed from ours, Leibowitz et al. found, as we did, that amount of separation influenced the induction effect when separations were smaller than $30^{\prime}$ of visual angle. Although induction effects were also visible at larger separations, there was practically no difference between separations of $30^{\prime}$ and $9^{\circ}$ visual angle.

It should be emphasized that robust induction of achromatic color is observed even when the two fields are widely separated. Both in our Experiment 2 and in Newson's (1958) experiments, the effect of the inducing field could be seen even at the widest separations; however, only with separations of less than about $30^{\prime}$ of visual angle did changes in the amount of separation substantially affect the results.

\section{EXPERIMENT 3}

Our third experiment had several purposes. First, we wanted to extend our findings regarding separation of ring and disk. Experiment 2 had shown little difference between color matches made with separations of $23^{\prime}$ and $52^{\prime}$ of visual angle. To confirm this result, in Experiment 3 we employed annuli of several widths, all greater than $30^{\prime}$ of visual angle. Our second aim was to study the effect of dichoptic presentation of the two luminances that interact to induce achromatic color. When two luminances are presented to different eyes, interaction at the retinal level is not possible. If color induction could be observed using a dichoptic mode of presentation, that induction would have to take place through postretinal mechanisms.

Additionally, we sought to determine whether the method we used to produce the annuli separating the disk 
and ring was critical. In Experiment 2, the different annuli were produced by varying the diameter of the disk. In the present experiment we produced annuli in two ways: by varying the disk and by varying the ring.

\section{Method}

Subjects. Another group of 8 undergraduate students participated as subjects.

Equipment and Procedure. The apparatus was modified to permit dichoptic presentation by introducing a series of mirrors that directed the image of the disk to the left eye only, while the right eye saw only the ring. These mirrors could be removed when binocular presentation was required. Two sets of three ring-and-disk displays were constructed. In one set (the variable-disk set) the dimensions of the ring were the same in each case; reductions in the size of the disk provided annuli of various widths separating the ring and the disk. The other set (the constant-area set) always used a disk of the same size. Separation was varied by increasing both the inner and outer diameters of the ring in such a way that the area of the ring also remained constant. Table 3 gives the dimensions of the stimuli. Luminances of the stimuli were the same as in the previous experiments.

In Experiment 3, the illumination in the Munsell box was spectrally different from the illumination used in Experiments 1 and 2 ; only fluorescent lamps were used, and pale yellow filters corrected the color of the light in the box so that its color temperature seemed the same as that of the larger box. In fact, Experiment 3 was done before Experiments 1 and 2, in which both fluorescent and incandescent lamps were used, because that made colortemperature matching easier. We did not foresee that the changed spectral composition of the light that was involved would interact with the Munsell chip display, but that was what apparently happened. The absorption spectra of the chips and of the paper that surrounded the chips were apparently different and caused them to reflect light of somewhat different luminances when the composition of the illumination was changed. This means that the matches obtained in the binocular condition in Experiment 3 cannot be compared with those from the other experiments.

Note that one stimulus pair was common to both the variabledisk and constant-area conditions. Thus there were actually five different ring-and-disk configurations, and each of these was presented both dichoptically and binocularly at each of three luminance ratios, resulting in 30 stimulus arrangements. The 8 subjects made color matches for each of these arrangements; as before, each subject made three matches for each arrangement, and his/her score was the average of the three.

Because changing the apparatus from the binocular to the dichoptic mode took time, the experiment was performed in two sessions. Half the subjects received the dichoptic mode in the first session, and the other half made binocular observations first. Order of presentation of the other variables was the same as in the previous ex-

Table 3

Dimensions of the Components of the Displays

\begin{tabular}{|c|c|c|c|c|}
\hline \multirow{2}{*}{$\begin{array}{c}\text { Disk } \\
\text { Diameter }(\mathrm{cm}) \\
\end{array}$} & \multicolumn{2}{|c|}{ Ring Diameter $(\mathrm{cm})$} & \multicolumn{2}{|c|}{ Annulus Width } \\
\hline & Inner & Outer & $\mathrm{cm}$ & $\operatorname{deg}$ \\
\hline \multicolumn{5}{|c|}{ Variable-Disk Condition } \\
\hline $\begin{array}{l}2.30 \\
3.18 \\
4.44\end{array}$ & $\begin{array}{l}5.70 \\
5.70 \\
5.70\end{array}$ & $\begin{array}{l}11.02 \\
11.02 \\
11.02\end{array}$ & $\begin{array}{l}1.70 \\
1.26 \\
0.63\end{array}$ & $\begin{array}{l}1.22 \\
0.90 \\
0.45\end{array}$ \\
\hline \multicolumn{5}{|c|}{ Constant-Area Condition } \\
\hline $\begin{array}{l}4.44 \\
4.44 \\
4.44\end{array}$ & $\begin{array}{l}6.84 \\
6.48 \\
5.70\end{array}$ & $\begin{array}{l}11.66 \\
11.46 \\
11.02\end{array}$ & $\begin{array}{l}1.20 \\
1.02 \\
0.63\end{array}$ & $\begin{array}{l}0.85 \\
0.72 \\
0.45\end{array}$ \\
\hline
\end{tabular}

Table 4

Mean Percent Reflectance of Matching Colors in the Variable-Disk Condition

\begin{tabular}{ccccc}
\hline & \multicolumn{3}{c}{ Luminance Ratio } & \\
\cline { 2 - 4 } Annulus Width & $13: 1$ & $8: 1$ & $3.75: 1$ & Average \\
\hline & \multicolumn{5}{c}{ Dichoptic Presentation } & \\
$0.63 \mathrm{~cm}$ & 58.5 & 62.9 & 67.2 & 63.0 \\
$1.26 \mathrm{~cm}$ & 57.3 & 61.2 & 66.8 & 61.8 \\
$1.76 \mathrm{~cm}$ & 56.7 & 61.4 & 66.4 & 61.5 \\
Average & 57.6 & 61.8 & 66.8 & \\
& & & & \\
$0.63 \mathrm{~cm}$ & 39.5 & 45.4 & 56.4 & 47.1 \\
$1.26 \mathrm{~cm}$ & 42.0 & 48.0 & 57.5 & 49.2 \\
$1.76 \mathrm{~cm}$ & 43.8 & 48.1 & 58.1 & 50.0 \\
Average & 41.8 & 47.2 & 57.3 & \\
\hline
\end{tabular}

Table 5

Mean Percent Reflectance of Matching Colors in the Constant-Area Condition

\begin{tabular}{ccccc}
\hline & \multicolumn{3}{c}{ Luminance Ratio } \\
\cline { 2 - 4 } Annulus Width & $13: 1$ & $8: 1$ & $3.75: 1$ & Average \\
\hline \multicolumn{5}{c}{ Dichoptic Presentation } \\
$0.63 \mathrm{~cm}$ & 58.8 & 62.9 & 56.4 & 63.0 \\
$1.02 \mathrm{~cm}$ & 56.8 & 62.0 & 59.7 & 62.5 \\
$1.20 \mathrm{~cm}$ & 55.8 & 60.6 & 57.5 & 61.1 \\
Average & 57.1 & 61.8 & 57.9 & \\
& & & & \\
$0.63 \mathrm{~cm}$ & 39.5 & 45.4 & 56.4 & 47.1 \\
$1.02 \mathrm{~cm}$ & 42.4 & 48.6 & 59.7 & 50.2 \\
$1.20 \mathrm{~cm}$ & 40.9 & 49.6 & 57.5 & 49.4 \\
Average & 40.9 & 47.9 & 57.9 & \\
\hline
\end{tabular}

periments, with order of ring-and-disk configurations randomized within subjects and order of luminance ratios randomized across subjects.

\section{Results and Discussion}

For purposes of analysis, the constant-area and variabledisk conditions were treated separately. Table 4 shows the results obtained in the variable-disk condition for both dichoptic and binocular observations. Statistical analysis of the data was performed by analysis of variance.

As in our earlier experiments, increases in the luminance ratio caused the induced color to appear darker. This effect was again statistically significant $[F(2,14)=$ $57.09, p<.001]$. Width of annulus, on the other hand, did not affect the appearance of the target field $[F(2,14)$ $=0.16, p>.05]$. Color matches were somewhat lighter under dichoptic than under binocular conditions; this effect was significant $[F(1,7)=8.37, p<.05]$. No interaction effects were significant for the variable-disk condition.

Table 5 shows the results for the constant-area condition. These data resemble very closely the data from the variable-disk condition presented in Table 4 . Here again, changes in luminance ratio significantly affected the apparent color $[F(2,14)=33.48, p<.001]$, width of an- 
nulus had no significant effect $[F(2,14)=2.94, p>.05]$, and color matches made in the dichoptic condition were again significantly lighter $[F(1,7)=8.50, p<.05]$.

One interaction effect was significant in the constantarea condition. The mode $\times$ ratio interaction yielded $F(2,14)=3.84, p<.05$. This reflects the fact that the color differences between dichoptic and binocular presentation become less pronounced as luminance ratios become less extreme. This tendency, which calls to mind the similar finding with regard to ring size in Expriment 1, is also present in the data from the variable-disk condition of Experiment 3; however, in that case it did not quite reach statistical significance $[F(2,14)=3.45, p>.05]$.

Two aspects of these data deserve emphasis. The first is that results from the constant-area and variable-disk conditions are virtually identical. This makes it unlikely that the absence of any substantial effect of increases in separation beyond $30^{\prime}$ of visual angle can be attributed to an interaction between amount of separation and relative areas of target and surround. Second, although the colors generated were somewhat lighter in the dichoptic condition, robust induction was still obtained. In the absence of induction, the target field would have appeared a luminous white, and the closest possible match on the Munsell chart would have been a chip of $90 \%$ reflectance. Instead, subjects consistently chose chips of a medium gray, and their choices were affected in the predicted way by changes in the luminance ratio, indicating that achromatic colors can be induced in the complete absence of retinal interaction.

\section{GENERAL DISCUSSION}

Five important findings emerge from these experiments. (1) Increasing the area of the surrounding luminance causes induced colors to appear darker; the extent of this effect grows as luminance ratio increases. (2) Separation of the two luminances causes induced colors to be lighter; amount of separation influences perceived color as long as the separation is less than approximately $30^{\prime}$ of visual angle. (3) At separations wider than $30^{\prime}$, induction of color continues to occur; however, the color perceived is not affected by the amount of separation over this range. (4) Achromatic colors can be induced with dichoptic presentation, in which only a single luminance is present on either retina. (5) Colors induced by dichoptic presentation of the two luminances are lighter than those perceived under comparable conditions with binocular presentation.

Returning to the question posed at the outset, we may examine these results to learn more about the level of processing at which induction takes place. Two possibilities were identified earlier: (1) very early (probably retinal) processes of the sort discussed by Cornsweet (1970); and (2) the more complex retinex (Land \& McCann, 1971), a conceptual mechanism whose physiological locus is not specified, which makes lightness comparisons across the entire visual field and evaluates them.
A retinal-process theory would easily accommodate our finding that separation between fields influences the resulting color when small separations are involved, since the lateral interaction upon which such a theory depends operates over limited retinal distances and is most powerful when fields are adjacent. The existence of color induction at wide separations is less easily explained by a retinal mechanism. Where the effect of the area of the surround is concerned, summation would account for an effect from within a limited area adjacent to the target, but not when the surround is larger. Thus, retinal summation could accommodate Newson's (1958) findings more readily than those from our Experiment 1 . Finally, the existence of dichoptically induced achromatic color cannot be accounted for by any mechanism operating at the retinal level.

The retinex model, with its focus on lightness relationships as they exist across the entire visual field, can deal with the existence of induction effects at wide separations. It also accommodates dichoptic induction, since the lightness comparisons presumably take place after input to the two eyes is combined. The retinex theory, as discussed by Land and McCann (1971), also emphasizes the role played by discontinuities in the field where areas of different luminance are adjacent; these discontinuities may explain the enhanced induction between luminances that are adjacent or separated by very small distances. However, the retinex theory does not predict the effect on induced color when the area of the inducing luminance changes. It is also difficult for the retinex theory to explain why the colors induced dichoptically should be lighter than those induced binocularly, because there are no provisions for the effect of neurological structure.

\section{REFERENCES}

Cole, R. E., \& Diamond, A. L. (1971). Amount of surround and testinducing separation in simultaneous brightness contrast. Perception \& Psychophysics, 9, 125-128.

CornsweEt, T. N. (1970). Visual perception. New York: Academic Press.

GelB, A. (1932). Die Erscheinungen des Simultan Kontrasts und der Eindruck der Feldbeleuchtung. Zeitschrift für Psychologie, 127, 42-59.

LAND, E. H., \& MCCANN, J. J. (1971). Lightness and the retinex the ory. Journal of the Philosophical Society of America, 61, 1-11.

Leibowitz, H., Mote, F. A., \& Thurlow, W. R. (1953). Simultaneous contrast as a function of separation between test and inducing fields. Journal of Experimental Psychology, 46, 453-456.

Newson, L. J. (1958). Some principles governing changes in the apparent lightness of test surfaces isolated from their normal backgrounds. Quarterly Journal of Experimental Psychology, 10, 82-95.

WALLACH, H. (1948). Brightness constancy and the nature of achromatic colors. Journal of Experimental Psychology, 38, 310-324.

\section{NOTE}

1. To enable the subject to fuse the two images, two short narrow vertical lines were centered above and below both the disk and the ring. When these lines were fused, the ring and disk appeared concentric in the combined display.

(Manuscript received March 9, 1987; revision accepted for publication October $5,1987$. ) 